\title{
Libpsht - algorithms for efficient spherical harmonic transforms
}

\author{
M. Reinecke
}

\author{
Max-Planck-Institut für Astrophysik, Karl-Schwarzschild-Str. 1, 85741 Garching, Germany \\ e-mail: martin@mpa-garching.mpg.de
}

Received 11 October 2010 / Accepted 1 November 2010

\begin{abstract}
Libpsht (or "library for performant spherical harmonic transforms") is a collection of algorithms for efficient conversion between spatial-domain and spectral-domain representations of data defined on the sphere. The package supports both transforms of scalars and spin- 1 and spin-2 quantities, and can be used for a wide range of pixelisations (including HEALPix, GLESP, and ECP). It will take advantage of hardware features such as multiple processor cores and floating-point vector operations, if available. Even without this additional acceleration, the employed algorithms are among the most efficient (in terms of CPU time, as well as memory consumption) currently being used in the astronomical community. The library is written in strictly standard-conforming C 90 , ensuring portability to many different hard- and software platforms, and allowing straightforward integration with codes written in various programming languages like $\mathrm{C}, \mathrm{C}++$, Fortran, Python etc. Libpsht is distributed under the terms of the GNU General Public License (GPL) version 2 and can be downloaded from http://sourceforge. net/projects/libpsht/.
\end{abstract}

Key words. methods: numerical - cosmic background radiation - large-scale structure of the Universe

\section{Introduction}

Spherical harmonic transforms (SHTs) have a wide range of applications in science and engineering. In the case of CMB science, which prompted the development of the library presented in this paper, they are an essential building block for extracting the cosmological power spectrum from full-sky maps, and are also used for generating synthesised sky maps in the context of Monte Carlo simulations. For all recent experiments in this field, the extraction of spherical harmonic coefficients has to be done up to very high multipole moments (up to $10^{4}$ ), which makes SHTs a fairly costly operation and therefore a natural candidate for optimisation. It also gives rise to a number of numerical complications that must be addressed by the transform algorithm.

The purpose of SHTs is the conversion between functions (or maps) on the sphere and their representation as a set of spherical harmonic coefficients in the spectral domain. In CMB science, such transforms are most often needed for quantities of spin 0 (i.e. quantities which are invariant with respect to rotation of the local coordinate system) and spin 2, because the unpolarised and polarised components of the microwave radiation are fields of these respective types. For applications concerned with gravitational lensing, SHTs of spins 1 and 3 are also sometimes required.

The paper is organised as follows. The following section lists the underlying equations anf the motivations for developing libpsht, as well as the goals of the implementation. A detailed explanation of the library's inner workings is presented in Sect. 3, and Sect. 4 contains a high-level overview of the interface it provides. Detailed studies regarding libpsht's performance, accuracy, and other quality indicators were performed, and their results presented in Sect. 5. Finally, a summary of the achieved (and not completely achieved) goals is given, together with an outlook on planned future extensions.

\section{Code capabilities}

\subsection{SHT equations}

A continuous spin- $s$ function $f(\vartheta, \varphi)$ with a spectral band limit of $l_{\max }$ is related to its corresponding set of spin spherical harmonic coefficients ${ }_{s} a_{l m}$ by the following equations:

$$
\begin{aligned}
f(\vartheta, \varphi) & =\sum_{m=-l_{\max }} \sum_{l=|m|}^{l_{\max }} a_{l m}{ }_{l m} Y_{l m}(\vartheta, \varphi) \\
{ }_{s} a_{l m} & =\int_{\vartheta=0}^{\pi} \int_{\varphi=0}^{2 \pi} \mathrm{d} \varphi \mathrm{d} \vartheta \sin \vartheta f(\vartheta, \varphi)_{s} Y_{l m}^{*}(\vartheta, \varphi) .
\end{aligned}
$$

Equations (1) and (2) are known as backward (or synthesis) and forward (or analysis) transforms, respectively. For a discretised spherical map consisting of a vector $\boldsymbol{p}$ of $N_{\text {pix }}$ pixels at locations $(\boldsymbol{\vartheta}, \varphi)$ and with (potentially weighted) solid angles $\boldsymbol{w}$, they change to:

$$
\begin{aligned}
\boldsymbol{p}_{n} & =\sum_{m=-l_{\max }}^{l_{\max }} \sum_{l=|m|}^{l_{\max }}{ }_{s} a_{l m} Y_{l m}\left(\boldsymbol{\vartheta}_{n}, \boldsymbol{\varphi}_{n}\right) \\
{ }_{s} \hat{a}_{l m} & =\sum_{n=1}^{N_{\mathrm{pix}}} \boldsymbol{p}_{n} \boldsymbol{w}_{n s} Y_{l m}^{*}\left(\boldsymbol{\vartheta}_{n}, \boldsymbol{\varphi}_{n}\right) .
\end{aligned}
$$

Depending on the choice of $l_{\max }, w$, and the grid geometry, the $\boldsymbol{p} \rightarrow{ }_{s} a_{l m}$ transform may only be approximate, which is indicated by choosing the identifier $\hat{a}$ instead of $a$.

The main purpose of the presented code is the efficient implementation (regarding CPU time as well as memory consumption) of Eqs. (3) and (4) for scalars as well as tensor quantities of spins \pm 1 and \pm 2 . 


\subsection{Design considerations}

At present, several SHT implementations are in use within the CMB community, like those distributed with the Fortran and $\mathrm{C}++$ implementations of HEALPix ${ }^{1}$ (Górski et al. 2005), GLESP $^{2}$ (Doroshkevich et al. 2005), s2hat ${ }^{3}$, spinsfast ${ }^{4}$ (Huffenberger \& Wandelt 2010), ccSHT $^{5}$, and IGLOO 6 (Crittenden \& Turok 1998); they offer varying degrees of performance, flexibility and feature completeness. The main design goal for libpsht was to provide a code which performs better than the SHTs in these packages and is versatile enough to serve as a drop-in replacement for them.

This immediately leads to several technical and pragmatic requirements which the code must fulfill:

Efficiency: the transforms provided by the library must perform at least as well (in terms of CPU time and memory) as those in the available packages, on the same hardware. If possible, all available hardware features (like multiple CPU cores, vector arithmetic instructions) should be used.

Flexibility: the library needs to support all of the above discretisations of the sphere; in addition, it should allow transforms of partial maps.

Portability: it must run on all platforms supported by the software packages above.

Universality: it must provide a clear interface that can be conveniently called from all the programming languages used for the packages above.

Completeness: if feasible, it should not depend on external libraries, because these complicate installation and handling by users.

Compactness: in order to simplify code development, maintenance and deployment, the library source code should be kept as small as possible without sacrificing readability.

It should be noted that the code was developed as a library for use within other scientific applications, not as a scientific application by itself. As such it strives to provide a comprehensive interface for programmers, rather than ready-made facilities for users.

\subsection{Supported discretisations}

For completely general discretisations of the sphere, the operation count for SHTs is $O\left(l_{\text {max }}^{2} N_{\text {pix }}\right)$ in both directions, which is not really affordable for today's resolution requirements. Fortunately, approaches of lower complexity exist for grids with the following geometrical constraints:

- the map pixels are arranged on $N_{\vartheta} \approx \sqrt{N_{\text {pix }}}$ iso-latitude rings, where the colatitude of each ring is denoted as $\boldsymbol{\vartheta}_{y}$;

- within one ring, pixels are equidistant in $\varphi$ and have identical weight $\boldsymbol{w}_{y}$; the number of pixels in each ring can vary and is denoted as $N_{\varphi, y}$, and the azimuth of the first pixel in the ring is $\varphi_{0, y}$.

\footnotetext{
${ }^{1}$ http://healpix.jpl.nasa.gov

2 http://glesp.nbi.dk

${ }^{3}$ http://www . apc . univ-paris7.fr/ radek/s2hat.html

${ }^{4}$ http://www.physics.miami.edu/ huffenbe/research/ spinsfast

5 http://crd.lbl.gov/ cmc/ccSHTlib/doc

${ }^{6}$ http://www. cita.utoronto.ca/ crittend/pixel.html
}

Under these assumptions, Eqs. (3) and (4) can be written as

$$
\begin{aligned}
\boldsymbol{p}_{x y} & =\sum_{m=-l_{\max }}^{l_{\max }} \sum_{l=|m|}^{l_{\max }}{ }_{s} a_{l m}{ }_{s} \lambda_{l m}\left(\boldsymbol{\vartheta}_{y}\right) \exp \left(\mathrm{i} m \varphi_{0, y}+\frac{2 \pi \mathrm{i} m x}{N_{\varphi, y}}\right) \\
{ }_{s} \hat{a}_{l m} & =\sum_{y=0}^{N_{\vartheta}-1} \sum_{x=0}^{N_{\varphi, y}-1} \boldsymbol{p}_{x y} \boldsymbol{w}_{y s} \lambda_{l m}\left(\boldsymbol{\vartheta}_{y}\right) \exp \left(-\mathrm{i} m \varphi_{0, y}-\frac{2 \pi \mathrm{i} m x}{N_{\varphi, y}}\right),
\end{aligned}
$$

where ${ }_{s} \lambda_{l m}(\vartheta):={ }_{s} Y_{l m}(\vartheta, 0)$. By subdividing both transforms into two stages

$$
\begin{aligned}
\boldsymbol{p}_{x y} & =\sum_{m=-l_{\max }}^{l_{\max }} F_{m, y} \exp \left(\mathrm{i} m \varphi_{0, y}+\frac{2 \pi \mathrm{i} m x}{N_{\varphi, y}}\right) \text { with } \\
F_{m, y} & :=\sum_{l=|m|}^{l_{\max }} a_{l m} \lambda_{l m}\left(\boldsymbol{\vartheta}_{y}\right), \quad \text { and } \\
{ }_{s} \hat{a}_{l m} & =\sum_{y=0}^{N_{y}-1} G_{m, y} \lambda_{l m}\left(\boldsymbol{\vartheta}_{y}\right) \quad \text { with } \\
G_{m, y} & :=\boldsymbol{w}_{y} \sum_{x=0}^{N_{\varphi, y}-1} \boldsymbol{p}_{x y} \exp \left(-\mathrm{i} m \varphi_{0, y}-\frac{2 \pi \mathrm{i} m x}{N_{\varphi, y}}\right)
\end{aligned}
$$

it becomes obvious that the steps ${ }_{s} a_{l m} \rightarrow F_{m, y}$ and $G_{m, y} \rightarrow{ }_{s} \hat{a}_{l m}$ require $O\left(N_{\vartheta} l_{\max }^{2}\right)$ operations, while a set of fast Fourier transforms - with the subdominant complexity of $O\left(N_{\vartheta} l_{\max } \log l_{\max }\right)-$ can be used for the steps $F_{m, y} \rightarrow \boldsymbol{p}_{x y}$ and $\boldsymbol{p}_{x y} \rightarrow G_{m, y}$, respectively. For any "useful" arrangement of the rings on the sphere, $N_{\vartheta}$ will be roughly proportional to $l_{\max }$, resulting in $O\left(l_{\max }^{3}\right)$ operations per SHT.

Practically all spherical grids which are currently used in the CMB field (HEALPix, ECP, GLESP, IGLOO) fall into this category, so libpsht can take advantage of this simplification. A notable exception is the "quadrilateral spherical cube" grid used by the COBE mission, but this is not really an issue, since COBE's resolution is so low that nowadays even brute-force SHTs produce results in acceptable time.

For the special case of the ECP grid, which is equidistant in both $\vartheta$ and $\varphi$, algorithms of the even lower complexity $O\left(l_{\max }^{2} \log ^{2} l_{\max }\right)$ have been presented (see, e.g., Driscoll \& Healy 1994; Wiaux et al. 2007). These were not considered for libpsht, because this would conflict with the flexibility goal. In addition, it has been observed that the constant prefactor for these methods is fairly high, so that they do not offer any performance advantage for small and intermediate $l_{\max }$; for transforms with high $l_{\max }$, on the other hand, their memory consumption is prohibitively high.

\subsection{Transformable quantities}

Without limiting the general applicability of the provided SHTs, libpsht imposes a few constraints on the format of the data it processes. For scalar transforms, it will only work with realvalued maps (the ubiquitous scenario in CMB physics); this is not really a limitation, since transforming a complex-valued map can be achieved by simply transforming its real and imaginary parts independently and computing a linear combination of the results.

For SHTs with $s \neq 0$, libpsht works on the so-called gradient (E) and curl (B) components of the tensor field

$$
\begin{aligned}
{ }_{|s|} E_{l m} & :=(-1)^{H} \frac{1}{2}\left(|s| a_{l m}+(-1)^{s}{ }_{-|s|} a_{l m}\right) \\
i_{|s|} B_{l m} & :=(-1)^{H} \frac{1}{2}\left({ }_{|s|} a_{l m}-(-1)^{s}{ }_{-|s|} a_{l m}\right)
\end{aligned}
$$


where ${ }_{|s|} E_{l m}^{*}=(-1)^{m}{ }_{|s|} E_{l,-m},{ }_{|s|} B_{l m}^{*}=(-1)^{m}{ }_{|s|} B_{l,-m}$ and $(-1)^{H}$ is a sign convention (Lewis 2005). The default setting for $H$ in libpsht is 1 , following the HEALPix convention, but this can be changed easily if necessary. On the real-space side, the tensor field is represented as two separate maps, containing the real and imaginary part, respectively.

In the following discussion, no fundamental distinction exists between ${ }_{|s|} E_{l m}$ and ${ }_{|s|} B_{l m}$, so for the sake of brevity both will be identified as ${ }_{s} a_{l m}$.

This choice of representation was made because it is compatible with the data formats used by most existing packages, which simplifies interfacing and data exchange. It also has the important advantage that any set of spherical harmonic coefficients is completely specified by its elements with $m>=0$, since ${ }_{s} a_{l m}$ and ${ }_{s} a_{l,-m}$ are coupled by symmetry relations.

\section{Algorithm description}

\subsection{Calculation of the spherical harmonic coefficients}

The central building block for every SHT implementation is the quick and accurate computation of spherical harmonics ${ }_{s} Y_{l m}(\vartheta, \varphi)$; for libpsht, these need only be evaluated at $\varphi=0$ and will be abbreviated as ${ }_{s} \lambda_{l m}(\vartheta)$.

\subsubsection{Scalar case}

For $s=0$, a proven method of determining the coefficients is using the recursion relation

$\lambda_{l m}=\cos \vartheta A_{l m} \lambda_{l-1, m}-B_{l m} \lambda_{l-2, m}$

with

$A_{l m}:=\sqrt{\frac{4 l^{2}-1}{l^{2}-m^{2}}} \quad$ and $\quad B_{l m}:=\frac{A_{l m}}{A_{l-1, m}}$

in the direction of increasing $l$; the starting values $\lambda_{m m}$ can be obtained from $\lambda_{00}=(4 \pi)^{-1 / 2}$ and the additional recursion relation

$\lambda_{m m}=-\sin \vartheta \sqrt{\frac{2 m+1}{2 m}} \lambda_{m-1, m-1}$

(Varshalovich et al. 1988). Since two values are required to start the recursion in $l$, one also uses $\lambda_{m+1, m}=\lambda_{m m} \sqrt{2 m+3}$.

Both recursions are numerically stable; nevertheless, their evaluation is not trivial, since the starting values $\lambda_{m m}$ can become extremely small for increasing $m$ and decreasing $\sin \vartheta$, such that they can no longer be represented by the IEEE754 number format used by virtually all of today's computers. This problem was addressed using the approach described by Prézeau \& Reinecke (2010), i.e. by augmenting the IEEE representation with an additional integer scale factor, which serves as an "enhanced exponent" and dramatically increases the dynamic range of representable numbers.

As is to be expected, this extended range comes at a somewhat higher computational cost; therefore the algorithm switches to standard IEEE numbers as soon as the $\lambda_{l m}$ have reached a region which can be safely represented without a scale factor. From that point on, the recursion in $l$ only requires three multiplications and one subtraction per $\lambda$ value, which are very cheap instructions on current microprocessors.

From the above it follows that, depending on the exact parameters of the SHT, a non-negligible fraction of the ${ }_{s} \lambda_{l m}(\vartheta)$ is extremely small, and that all terms in Eqs. (8) and (9) containing such values as a factor do not contribute in a measurable way to the SHT's result, due to the limited dynamic range of the floating-point format in which the result is produced. For that reason, libpsht records the index $l_{\text {th }}$, at which the $\left.\right|_{s} \lambda_{l m}(\vartheta) \mid$ first exceed a threshold $\varepsilon_{\text {th }}$ (which is set to the conservative value of $10^{-30}$ ) during every $l$ recursion. The remaining operations will then be carried out for $l \geq l_{\text {th }}$ only.

Another acceleration is achieved by skipping the $l$-recursion for an $(m, \vartheta)$-tuple entirely, if another recursion was performed before with $m_{\mathrm{c}} \leq m$ and $\left|\cos \vartheta_{\mathrm{c}}\right| \leq|\cos \vartheta|<1$ that never crossed $\varepsilon_{\text {th }}$.

In the case that a ring at colatitude $\vartheta$ has a counterpart at $\pi-\vartheta$ (which is almost always the case for the popular grids), the ${ }_{s} \lambda_{l m}(\vartheta)$ recursion need only be performed for one of those; the values for the other ring are quickly obtained by the symmetry relation

${ }_{s} \lambda_{l m}(\pi-\vartheta)=(-1)^{l-s}{ }_{-s} \lambda_{l m}(\vartheta)$.

Libpsht will identify such ring pairs automatically and treat them in an optimised fashion.

\subsubsection{Tensor case}

Following Lewis (2005), libpsht does not literally compute the tensor harmonics ${ }_{ \pm s} \lambda_{l m}$, but rather their linear combinations

${ }_{s} F_{l m}^{ \pm}:=\frac{1}{2}\left({ }_{+s} \lambda_{l m} \pm(-1)^{s}{ }_{-s} \lambda_{l m}\right)$.

These are not directly obtained using a recursion, but rather by first computing the spin- $0 \lambda_{l m}$ and subsequently applying spin raising and lowering operators; see Appendix A of Lewis (2005) for the relevant formulae. Whenever scalar and tensor SHTs are performed simultaneously, this has the advantage that the scalar $\lambda_{l m}$ can be re-used for both; in CMB science this scenario is very common, since any transform involving a polarised sky map requires SHTs for both $s=0$ and $s= \pm 2$.

The approach can be extended to higher spins in principle, but for $|s|>2$ the expressions become quite involved and are also slow to compute. For this reason, libpsht's transforms are currently limited to $s=0, \pm 1, \pm 2$. Should a need for SHTs at higher $s$ become evident, a method making use of the relationships between ${ }_{s} \lambda_{l m}$ and the Wigner $d_{m m^{\prime}}^{l}$ matrix elements seems most promising, since they obey very similar recursion relations (see, e.g., Kostelec \& Rockmore 2008), for which a recent implementation is available (Prézeau \& Reinecke 2010).

\subsubsection{Re-using the computed ${ }_{s} \lambda_{l m}(\vartheta)$}

Many packages allow the user to supply an array containing precomputed ${ }_{s} \lambda_{l m}(\vartheta)$ coefficients to the SHT routines, which can speed up the computation by a factor of up to $\approx 2$. Libpsht does not provide such an option; this may seem surprising at first, but it was left out deliberately for the following reasons:

- Even if supplied externally, the coefficients have to be obtained by some method. Reading them from mass storage is definitely not a good choice, since they can be computed onthe-fly much more quickly on current hardware. Externally provided values can therefore only provide a benefit if they are used in at least two consecutive SHTs.

- When using multiple threads in combination with precomputed ${ }_{s} \lambda_{l m}(\vartheta)$, computation time soon becomes dominated by memory access operations to the table of coefficients, because the memory bandwidth of a computer does not scale with the number of processor cores. This will severely limit 
the scalability of the code. It is safe to assume that this problem will become even more pronounced in the future, since CPU power tends to grow more rapidly than memory bandwidth (Drepper 2007).

- For problem sizes with $l_{\max } \gtrsim 2000$, when additional performance would be most desirable, the precomputed tables (whose size is proportional to $N_{\vartheta} l_{\text {max }}^{2}$ ) become so large that they no longer fit into main memory. A conservative estimate for the size of the ${ }_{s} \lambda_{l m}(\vartheta)$ array at $l_{\max }=2000$ and $s=0$ is about $8 \mathrm{~GB}$; the Fortran HEALPix library currently requires $32 \mathrm{~GB}$ in this situation.

- Libpsht supports multiple simultaneous SHTs, and will compute the ${ }_{s} \lambda_{l m}(\vartheta)$ only once during such an operation, reusing them for all individual transforms. Since this is done in a piece-by-piece fashion (see Sect. 3.3), only small subsets of size $O\left(l_{\max }\right)$ need to be stored, which is more or less negligible; this approach also implies that the internally precomputed ${ }_{s} \lambda_{l m}(\vartheta)$ subsets will most likely remain in the CPU cache and therefore not be subject to the memory bandwidth limit.

\subsection{Fast Fourier transform}

Libpsht uses an adapted version of the well-known fftpack library (Swarztrauber 1982), ported to the C language (with a few minor adjustments) by the author. This package performs well for FFTs of lengths $N$ whose prime decomposition only contains the factors 2,3 , and 5 . For prime $N$, its complexity degrades to $O\left(N^{2}\right)$, which should be avoided, so for $N$ containing large prime factors, Bluestein's algorithm (Bluestein 1968) is employed, which allows replacing an FFT of length $N$ by a convolution (conveniently implemented as a multiplication in Fourier space) of any length $\geq 2 N-1$. By appropriate choice of the new length, the desired $O(N \log N)$ complexity can be reestablished, at the cost of a higher prefactor.

Obviously it would have been possible to use the very popular and efficient FFTW ${ }^{7}$ library (Frigo \& Johnson 2005) for the Fourier transforms, but since this package is fairly heavyweight, this would conflict with libpsht's completeness or compactness goals. Even though libfftpack with the Bluestein modification will be somewhat slower than FFTW, the efficiency goal is not really compromised: libpsht's algorithms have an overall complexity of $O\left(N_{\vartheta} l_{\max }^{2}\right)$, so that the FFT part with its total complexity of $O\left(N_{\vartheta} l_{\max } \log l_{\max }\right)$ will (for nontrivial problem sizes) never dominate the total CPU time.

\subsection{Loop structure of the SHT}

As Eqs. (5) and (6) and the complexity of $O\left(N_{\vartheta} l_{\max }^{2}\right)$ already suggest, a code implementing SHTs on an iso-latitude grid will contain at least three nested loops which iterate on $y, m$, and $l$, respectively. The overall speed and/or memory consumption of the final implementation depend sensitively on the order in which these loops are nested, since different hierarchies are not equally well suited for precomputation of common quantities used in the innermost loop.

The choice made for the computation of the ${ }_{s} \lambda_{l m}(\vartheta)$, i.e. to obtain them by $l$-recursion (see Sect. 3.1) strongly favors placing the loop over $l$ innermost; so only the order of the $y$ and $m$ loops remains to be determined.

From the point of view of speed optimisation, arguably the best approach would be to iterate over $m$ in the outermost loop,

\footnotetext{
${ }^{7}$ http://fftw.org
}

then over the ring number $y$, and finally over $l$. This allows precomputing the $A_{l m}$ and $B_{l m}$ quantities (see Sect. 3.1) exactly once and in small chunks, for an additional memory cost of only $O\left(l_{\max }\right)$. However, this method requires additional $O\left(N_{\vartheta} l_{\max }\right)$ storage for the intermediate products $F_{m, y}$ or $G_{m, y}$, which is comparable to the size of the input and output data. This was considered too wasteful, since it prohibits performing SHTs whose input and output data already occupy most of the available computer memory.

Unfortunately, switching the order of the two outermost loops does not improve the situation: now, one is presented with the choice of either precomputing the full two-dimensional $A_{l m}$ and $B_{l m}$ arrays, which is not acceptable for the same reason that prohibits storing the entire $F_{m, y}$ and $G_{m, y}$ arrays, or recomputing their values over and over, which consumes too much CPU time.

To solve this dilemma, it was decided not to perform the SHTs for a whole map in one go, but rather to subdivide them into a number of partial transforms, each of which only deals with a subset of rings (or ring pairs). An appropriate choice for the number of parts will result in near-optimal performance combined with only small memory overhead; the most symmetrical choice of $N_{\vartheta}^{1 / 2}$ leads to a time overhead of $O\left(N_{\vartheta}^{1 / 2} l_{\max }^{2}\right)$ and additional memory consumption of $O\left(N_{\vartheta}^{1 / 2} l_{\max }\right)$, both of which are small compared to the total CPU and storage requirements. Libpsht uses sub-maps of roughly 100 or $N_{\vartheta} / 10$ rings (or ring pairs), whichever is larger.

The arrangement described above is sufficient for a single SHT. Since libpsht supports multiple simultaneous SHTs, another loop hierarchy had to be introduced. A pseudo-code listing illustrating the complete design of the SHT algorithm (including the subdominant parts performing the FFTs) is presented in Fig. 1.

\subsection{Further acceleration techniques}

To make use of multiple CPU cores, if available, the algorithm's loop over $m$, as well as the code blocks performing the FFTs, have been parallelised using OpenMP ${ }^{8}$ directives, requesting dynamic scheduling for every iteration; this is indicated by the "OpenMP" tag in Fig. 1. As long as the SHTs are large enough, this results in very good scaling with the number of cores available (see also Sect. 5.2.4).

If the target machine supports the SSE2 instruction set ${ }^{9}$ (which is the case for all AMD/Intel CPUs introduced since around 2003), its ability to perform two arithmetic operations of the same kind with a single machine instruction is used to process two ring (pairs) simultaneously, greatly improving the overall execution speed. The relevant loop is marked with "SSE2" in Fig. 1. The necessary changes are straightforward for the most part; however, special attention must be paid to the $l$ recursion, because the two sequences of ${ }_{s} \lambda_{l m}(\vartheta)$ will cross the threshold to IEEE-representable numbers at different $l_{\text {th }}$. Fortunately this complication can be addressed without a significant slowdown of the algorithm.

The data types used and the functions called in order to use the SSE2 instruction set are not part of the C90 language standard, and will therefore not be known to all compilers and on all hardware platforms. However there is a portable way to detect compiler and hardware support for SSE2; if the result of this check is negative, only the standard-compliant floating-point

\footnotetext{
8 http://openmp.org

9 http://en.wikipedia.org/wiki/SSE2
} 


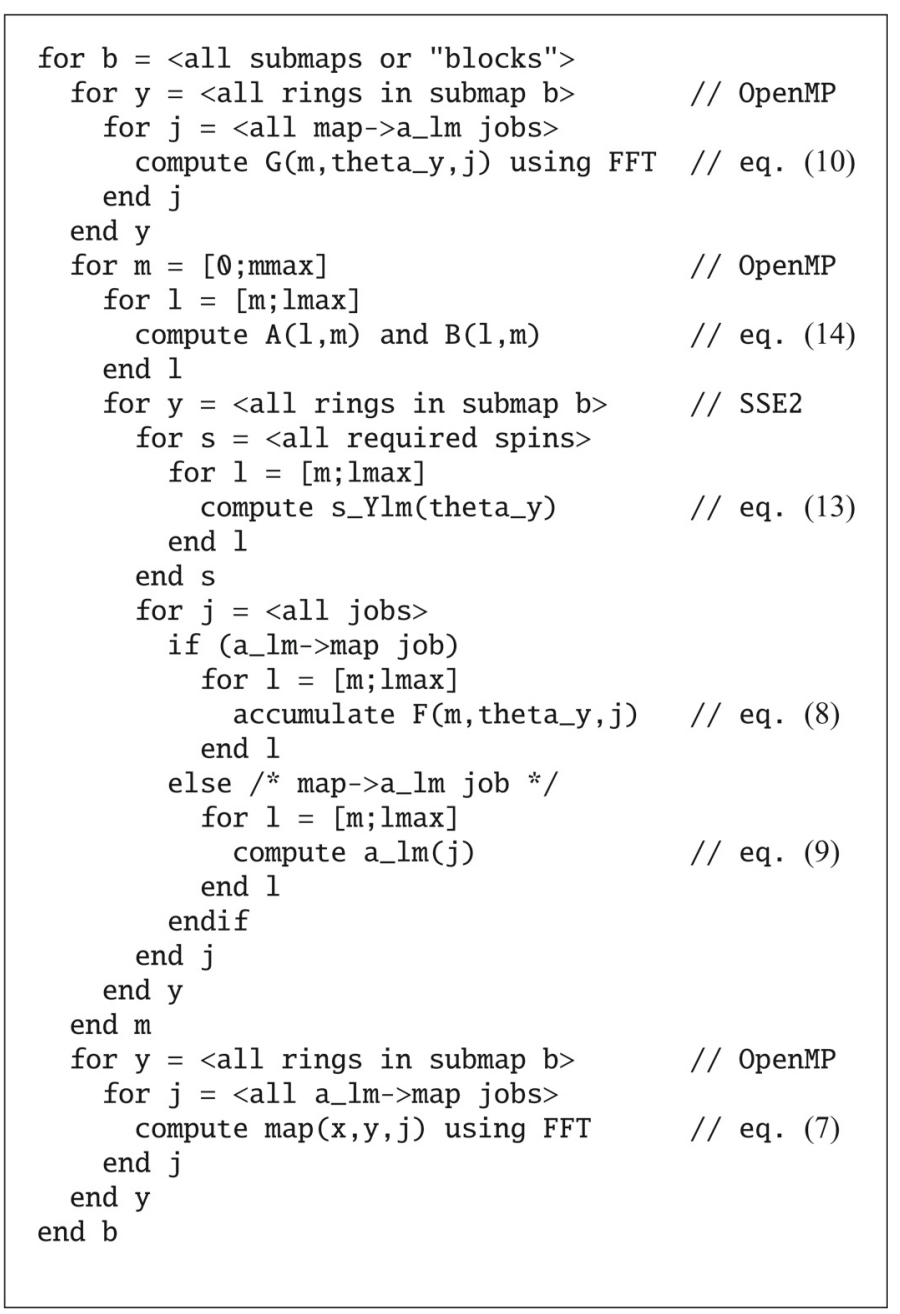

Fig. 1. Schematic loop structure of libpsht's transform algorithm.

implementation of libpsht will be compiled, without the necessity of user intervention.

Similarly, the OpenMP functionality is accessed using socalled \#pragma compiler directives, which are simply ignored by compilers lacking the required capability, reducing the source code to a single-threaded version.

Both extensions are supported by the widely used gcc $^{10}$ and Intel compilers.

\section{Programming interface}

Only a high-level overview of the provided functionality can be given here. For the level of detail necessary to actually interface with the library, the reader is referred to the technical documentation provided alongside the source code.

\subsection{Description of input/output data}

Due to libpsht's universality goal, it must be able to accept input data and produce output data in a wide variety of storage schemes, and therefore only makes few assumptions about how maps and $a_{l m}$ are arranged in memory.

\footnotetext{
${ }^{10}$ http://gcc.gnu.org
}

A tesselation of the sphere and the relative location of its pixels in memory is described to libpsht by the following set of data:

- the total number of rings in the map; and

- for every ring

- the number of pixels $N_{\varphi, y}$;

- the index of the first pixel of the ring in the map array;

- the stride between indices of consecutive pixels in this ring;

- the azimuth of the first pixel $\varphi_{0, y}$;

- the colatitude $\boldsymbol{\vartheta}_{y}$;

- the weight factor $\boldsymbol{w}_{y}$. This is only used for forward SHTs and is typically the solid angle of a pixel in this ring.

For $a_{l m}$, less information is necessary:

- the maximum $l$ and $m$ quantum numbers available in the set;

- the index stride between $a_{l m}$ and $a_{l+1, m}$;

- an integer array containing the (hypothetical) index of the element $a_{0, m}$.

Due to the convention described in Sect. 2.4, only coefficients with $m \geq 0$ will be accessed.

These two descriptions are flexible enough to describe all spherical grids of interest, as well as all data storage schemes that are in widespread use within the CMB community. In combination with a data pointer to the first element of a map or set of $a_{l m}$, they describe all characteristics of a set of input or output data that libpsht needs to be aware of.

It should be noted that the map description trivially supports transforms on grids that only cover parts of the $\vartheta$ range; rings that should not be included in map synthesis or analysis can simply be omitted in the arrays describing the grid geometry, speeding up the SHTs on this particular grid. More general masks, which affect parts of rings, have to be applied by explicitly setting the respective map pixels to zero before map analysis or after map synthesis.

Libpsht will only work on data that is already laid out in main memory according to the structures mentioned above. There is no functionality to access data on mass storage directly; this task must be undertaken by the programs calling libpsht functionality. Given the bewildering variety of formats which are used for storing spherical maps and $a_{l m}$ on disk - and with the prospect of these formats changing more or less rapidly in the future $-\mathrm{I} / \mathrm{O}$ was not considered a core functionality of the library.

\subsection{Supported floating-point formats}

In addition to the flexibility in memory ordering, libpsht can also work on data in single- or double-precision format, with the requirement that all inputs and outputs for any set of simultaneous transforms must be of the same type. Internally, however, all computations are performed using double-precision numbers, so that no performance gain can be achieved by using singleprecision input and output data.

\subsection{Multiple simultaneous SHTs}

As already mentioned, it is possible to compute several SHTs at once, independent of direction and spin, as long as their map and $a_{l m}$ storage schemes described above are identical. This is achieved by repeatedly adding SHT requests, complete with pointers to their input and output arrays, to a "job list", and finally calling the main SHT function, providing the job list, map information and $a_{l m}$ information as arguments. 


\subsection{Calling the library from other languages}

Due to the choice of $\mathrm{C}$ as libpsht's implementation language, the library can be interfaced without any complications from other codes written in $\mathrm{C}$ or $\mathrm{C}++$. Using the library from languages like Python and Java should also be straightforward, but requires some glue code.

For Fortran the situation is unfortunately a bit more complicated, because a well-defined interface between Fortran and C has only been introduced with the Fortran2003 standard, which is not yet universally supported by compilers. Due to the philosophy of incrementally building a job list and finally executing it (see Sect. 4.3), simply wrapping the $\mathrm{C}$ functions using cfortran. $\mathrm{h}^{11}$ glue code does not work reliably, so that building a portable interface is a nontrivial task. Nevertheless, the Fortran90 code beam $2 \mathrm{alm}$ of the Planck mission's simulation package has been successfully interfaced with libpsht.

\section{Benchmarks}

In order to evaluate the reliability and efficiency of the library, a series of tests and comparisons with existing implementations were performed. All of these experiments were executed on a computer equipped with $64 \mathrm{~GB}$ of main memory and Xeon E7450 processors (24 CPU cores overall) running at $2.4 \mathrm{GHz}$. gcc version 4.4.2 was used for compilation.

\subsection{Correctness and accuracy}

The validation of libpsht's algorithms was performed in two stages: first, the result of a backward transform was compared to that produced with another SHT library using the same input, and afterwards it was verified that a pair of backward and forward SHTs reproduces the original $a_{l m}$ with sufficient precision.

All calculations in this section start out with a set of ${ }_{s} a_{l m}$ that consists entirely of uniformly distributed random numbers in the range $(-1 ; 1)$, except for the imaginary parts of the ${ }_{s} a_{l, 0}$, which must be zero for symmetry reasons, and coefficients with $l<|s|$, which have no meaning in a spin- $s$ transform and are set to zero as well.

\subsubsection{Validation against other implementations}

For this test, a set of $a_{l m}$ with $l_{\max }=2048$ was generated using the above prescription and converted to a $N_{\text {side }}=1024$ HEALPix map by both libpsht and the Fortran HEALPix facility synfast. This was done for spins 0 and 2 , mimicking the synthesis of a polarised sky map. Comparison of the resulting maps showed a maximum discrepancy between individual pixels of $1.55 \times 10^{-7}$, and the RMS error was $6.3 \times 10^{-13}$, which indicates a very good agreement.

\subsubsection{Accuracy of recovered $\hat{a}_{l m}$}

An individual accuracy test consists of a map synthesis, followed by an analysis of the obtained map, producing the result ${ }_{s} \hat{a}_{l m}$.

Two quantities are used to assess the agreement between these two sets of spherical harmonic coefficients; they are defined as

$$
\begin{aligned}
\varepsilon_{\mathrm{rms}} & :=\sqrt{\frac{\left.\sum_{l m}\right|_{s} a_{l m}-\left.{ }_{s} \hat{a}_{l m}\right|^{2}}{\sum_{l m}\left|{ }_{s} a_{l m}\right|^{2}}} \text { and } \\
\varepsilon_{\max }: & :=\max _{l m}\left(\left|\mathfrak{R}\left({ }_{s} a_{l m}-{ }_{s} \hat{a}_{l m}\right)\right|,\left|\mathfrak{J}\left({ }_{s} a_{l m}-{ }_{s} \hat{a}_{l m}\right)\right|\right) .
\end{aligned}
$$

\footnotetext{
11 http://www-zeus.desy.de/ burow/cfortran/
}

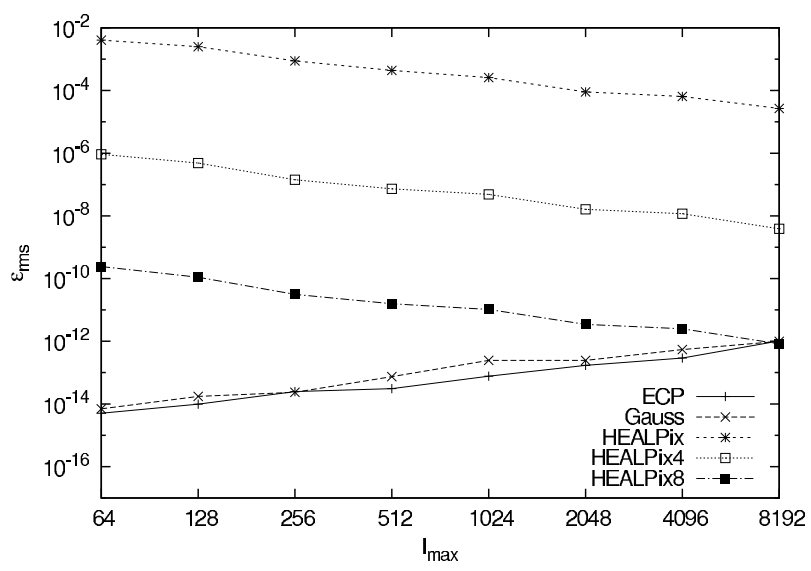

Fig. 2. $\varepsilon_{\text {rms }}$ for libpsht transform pairs on ECP, Gaussian and HEALPix grids for various $l_{\max }$. Every data point is the maximum error value encountered in transform pairs of all supported spins. "Healpix4" and "Healpix8" refer to iterative analyses with 4 and 8 steps, respectively.

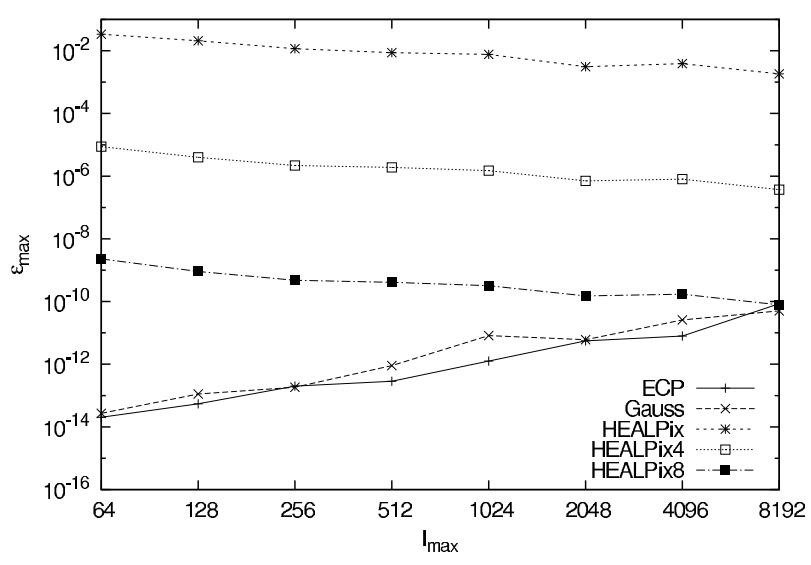

Fig. 3. $\varepsilon_{\max }$ for libpsht transform pairs on ECP, Gaussian and HEALPix grids for various $l_{\max }$. Every data point is the maximum error value encountered in transform pairs of all supported spins. "Healpix4" and "Healpix8" refer to iterative analyses with 4 and 8 steps, respectively.

A variety of such tests was performed for different grid geometries, $l_{\max }$, and spins; the results are shown in Figs. 2 and 3.

For ECP and "Gaussian" grids, each ring consisted of $2 l_{\max }+$ 1 pixels, and the grids contained $2 l_{\max }+2$ and $l_{\max }+1$ rings, respectively. The $\boldsymbol{\vartheta}_{y}$ for the Gaussian grid were chosen to coincide with the roots of the Legendre polynomial $P_{l_{\max }+1}(\vartheta)$. Under these conditions, and with appropriately chosen pixel weights (taken from Driscoll \& Healy 1994; and Doroshkevich et al. $2005)$, the transform pair should reproduce the original $a_{l m}$ exactly, were it not for the limited precision of floating-point arithmetics. In fact, the errors measured for SHTs on those two grids are extremely small and close to the theoretical limit.

For the transform pairs on HEALPix grids, a resolution parameter of $N_{\text {side }}=l_{\max } / 2$ was chosen. Here, the recovered ${ }_{s} \hat{a}_{l m}$ will only be an approximation of the original ${ }_{s} a_{l m}$; if higher accuracy is required, this approximation can be improved by Jacobi iteration, as described in the HEALPix documentation. The figures show clearly that $\varepsilon_{\text {rms }}$ is around $10^{-3}$ for the simple transform pair and can be reduced dramatically by iterated analysis. 
M. Reinecke: Libpsht - algorithms for efficient spherical harmonic transforms

Table 1. Single-core CPU time for various SHTs with $l_{\max }=2048$.

\begin{tabular}{lcccc}
\hline \hline Direction & Spin & ECP & Gaussian & HEALPix \\
\hline forward & 0 & $15.14 \mathrm{~s}$ & $7.89 \mathrm{~s}$ & $13.11 \mathrm{~s}$ \\
backward & 0 & $14.74 \mathrm{~s}$ & $7.53 \mathrm{~s}$ & $12.65 \mathrm{~s}$ \\
forward & 1 & $29.49 \mathrm{~s}$ & $15.36 \mathrm{~s}$ & $25.05 \mathrm{~s}$ \\
backward & 1 & $27.83 \mathrm{~s}$ & $14.30 \mathrm{~s}$ & $23.85 \mathrm{~s}$ \\
forward & 2 & $31.91 \mathrm{~s}$ & $16.91 \mathrm{~s}$ & $28.15 \mathrm{~s}$ \\
backward & 2 & $30.33 \mathrm{~s}$ & $15.60 \mathrm{~s}$ & $26.51 \mathrm{~s}$ \\
\hline
\end{tabular}

Notes. The ECP grid had $2 l_{\max }+1$ pixels per ring and $2 l_{\max }+2$ rings, the Gaussian grid had $2 l_{\max }+1$ pixels per ring and $l_{\max }+1$ rings, and the $N_{\text {side }}$ parameter of the HEALPix grid was $l_{\max } / 2$.

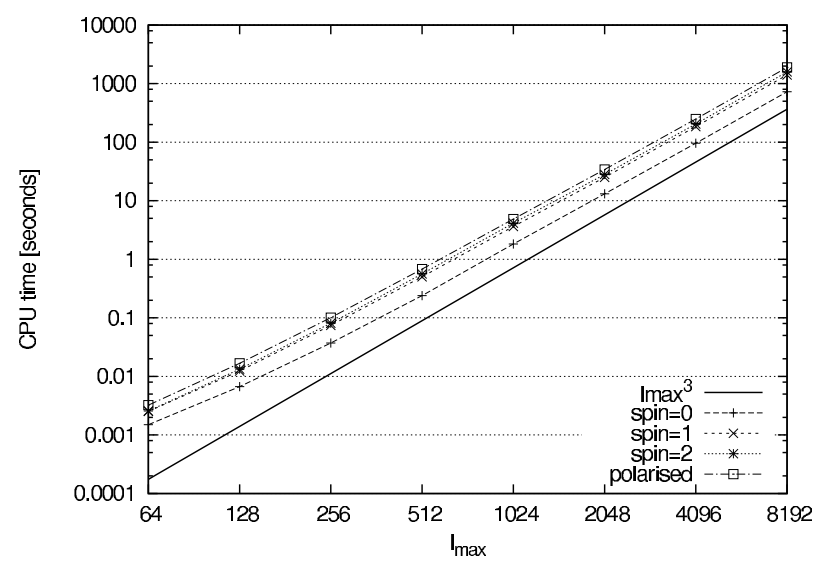

Fig. 4. Timings of single-core forward SHTs on a HEALPix grid with $N_{\text {side }}=l_{\max } / 2$. The annotation "polarised" refers to a combined SHT of spin 0 and 2 quantities, which is a very common case in CMB physics.

\subsection{Time consumption}

\subsubsection{Relative cost of SHTs}

Table 1 shows the single-core CPU times for SHTs of different directions, spins and grids, but with identical band limit, in order to illustrate their relative cost. It is fairly evident that forward and backward SHTs with otherwise identical parameters require almost identical time, with the $a_{l m} \rightarrow$ map direction being slightly slower. Since this relation also holds for other band limits, only the timings for one direction will be shown in the following figures, in order to reduce clutter.

SHTs with $|s|>0$ take roughly twice the time of corresponding scalar ones; here the $s=2$ case is slightly more expensive than $s=1$, because computing the ${ }_{2} F_{l m}^{ \pm}$from $\lambda_{l m}$ requires more operations than computing the ${ }_{1} F_{l m}^{ \pm}$.

The timings also indicate the scaling of the SHT cost with $N_{\vartheta}$, which is practically equal for HEALPix and ECP grids, but half as high for the Gaussian grid.

These timings, as well as all other timing results for libpsht in this paper, were obtained using SSE2-accelerated code. Switching off this feature will result in significantly longer execution times; on the benchmark computer the factor lies in the range of 1.6 to 1.8 .

\subsubsection{Scaling with $I_{\max }$}

SHTs of different spins and a wide range of band limits were performed on a single CPU core, in order to assess the correlation between CPU time and $l_{\max }$; the results are shown in Fig. 4. As expected, the time consumption scales almost with $l_{\text {max }}^{3}$; deviations from this behaviour only become apparent for lower band

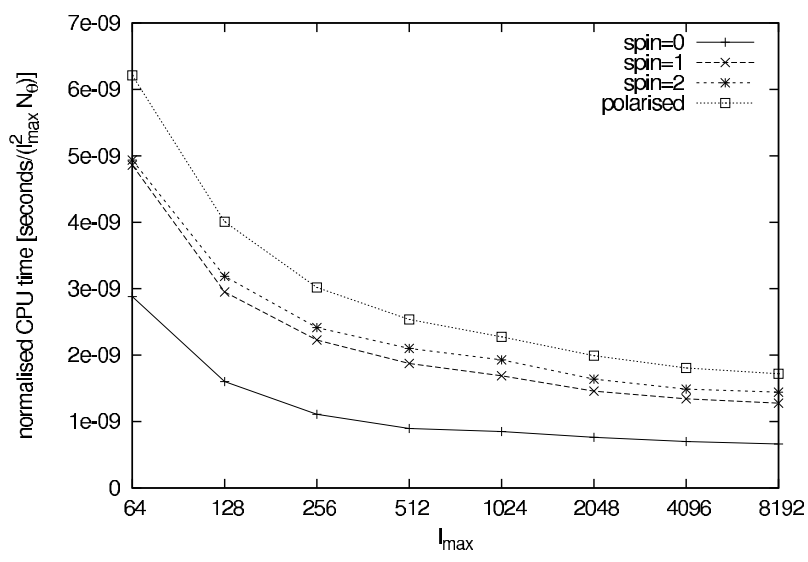

Fig. 5. Same as Fig. 4, but with CPU time divided by $l_{\max }^{2} N_{\vartheta}$.

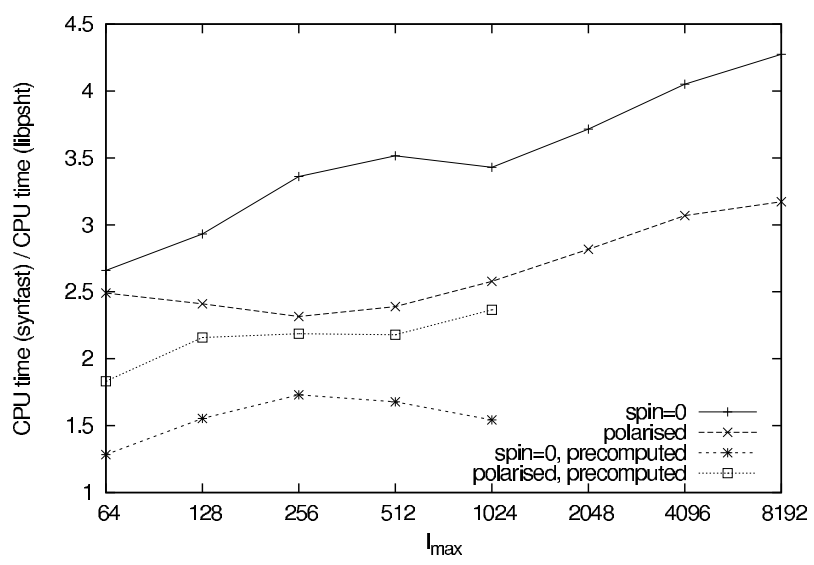

Fig. 6. CPU time ratios between the Fortran HEALPix synfast code and libpsht for various backward SHTs on a HEALPix grid with $N_{\text {side }}=l_{\max } / 2$.

limits, where SHT components of lower overall complexity (like the FFTs, computation of the $A_{l m}$ and $B_{l m}$ coefficients and initialisation of data structures) are no longer negligible. Switching to a normalised representation of the CPU time, as was done in Fig. 5, makes this effect much more evident; even for transforms of very high band limit the curves are not entirely horizontal, which would indicate a pure $O\left(l_{\max }^{2} N_{\vartheta}\right)$ algorithm.

\subsubsection{Performance comparison with Fortran HEALPix}

Figure 6 shows the ratio of CPU times for equivalent SHTs carried out using the synfast facility of Fortran HEALPix and libpsht, using identical hardware resources. It allows several deductions:

- in all tested scenarios, libpsht's implementation is significantly faster, even when precomputed coefficients are used with synfast;

- the performance gap is markedly wider for $s=0$ transforms, which indicates that libpsht's $l$-recursion is the part which has been accelerated by the largest factor in comparison to the Fortran HEALPix SHT library;

- the polarised synfast SHTs only show a marginal speed improvement when using precomputed coefficients; for the scalar transforms, the difference is much more noticeable. Although this observation has no direct connection to libpsht, it is nevertheless of interest: most likely the missing acceleration is caused by saturation of the memory 


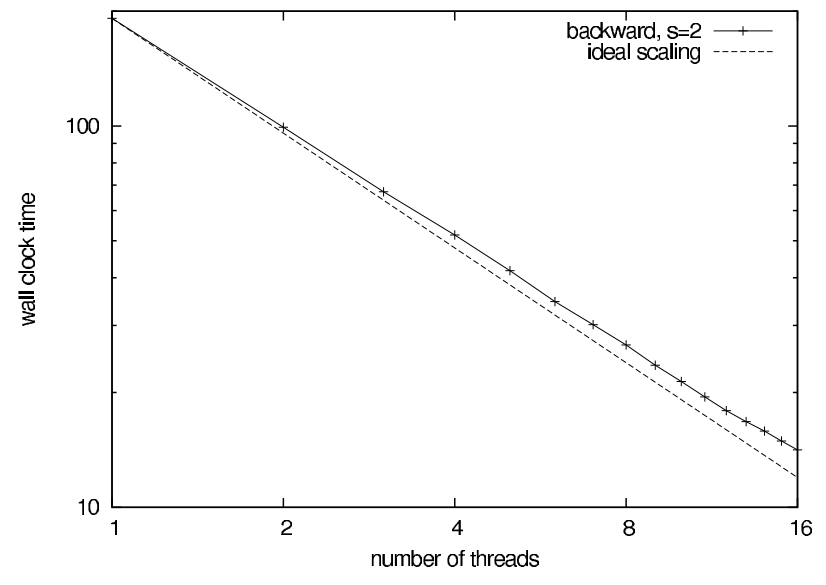

Fig. 7. Elapsed wall clock time for a backward SHT with $s=2, l_{\max }=$ 4096 , and $N_{\text {side }}=2048$ on a HEALPix grid, run with a varying number of OpenMP threads.

bandwidth, as discussed in Sect. 3.1.3, and confirms the decision not to introduce such a feature into libpsht.

\subsubsection{Scaling with number of cores}

As illustrated in Fig. 1, all CPU-intensive parts of libpsht's transform algorithm are instrumented for parallel execution on multicore computers using OpenMP directives. Figure 7 shows the wall clock times measured for a single SHT that was run using a varying number of OpenMP threads. The maximum number of threads was limited to 16 (in contrast to the maximum useful value of 24 on the benchmark computer), because the machine was not completely idle during the libpsht performance measurements, and the scaling behaviour at larger numbers of threads would have been influenced by other running tasks.

It is evident that the overhead due to workload distribution among several threads is quite small: for the 16-processor run, the accumulated wall clock time is only $18 \%$ higher than for the single-processor SHT. An analogous experiment was performed with the Fortran HEALPix code synfast; here the overhead for 16 threads was around $35 \%$.

\subsubsection{Comparison with theoretical CPU performance}

For this experiment, the library's code was enhanced to count the number of floating-point operations that were carried out during the SHT. This instrumentation was only done in the parts of the code with $O\left(l_{\max }^{2} N_{\vartheta}\right)$ complexity, i.e. everything related to creation and usage of the ${ }_{s} \lambda_{l m}(\vartheta)$. Operations that were necessary only for the purpose of numerical stability (e.g. due to the rangeextended floating-point format) were not taken into account. Dividing the resulting number of operations by the CPU time for a single-core uninstrumented run of the same SHT provides a conservative estimate for the overall floating-point performance of the algorithm. Measuring $s=0$ and $s=2 a_{l m} \rightarrow$ map SHTs on a HEALPix grid with $N_{\text {side }}=1024$ and $l_{\max }=2048$ resulted in $2.1 \mathrm{GFlops} / \mathrm{s}$ and $4.1 \mathrm{GFlops} / \mathrm{s}$, respectively. This corresponds to 0.88 and 1.71 floating-point operations per clock cycle, or $22 \%$ and $43 \%$ of the theoretical peak performance. Both of these percentages are quite high for an implementation of a nontrivial algorithm, which can be seen as a hint that there is not much more room for further optimisation on this hardware architecture. In other words, further speedups can only be achieved by a fundamental change in the underlying algorithm.
Table 2. Speedup for simultaneous vs. consecutive SHTs on a HEALPix grid with $N_{\text {side }}=1024$ and $l_{\max }=2048$.

\begin{tabular}{lrrr}
\hline \hline Transforms & $T_{\text {simul }} / \mathrm{s}$ & $T_{\text {separate }} / \mathrm{s}$ & Speedup \\
\hline 1a0 & 12.65 & 12.65 & 1.00 \\
2a0 & 18.06 & 25.30 & 1.40 \\
3a0 & 23.47 & 37.95 & 1.62 \\
$5 \mathrm{a} 0$ & 34.77 & 63.25 & 1.82 \\
$10 \mathrm{a} 0$ & 62.56 & 126.50 & 2.02 \\
3a0+3m0 & 41.72 & 77.46 & 1.85 \\
1a0+1a1+1a2+1m0+1m1+1m2 & 78.79 & 129.44 & 1.64 \\
$4 \mathrm{~m} 2$ & 63.68 & 112.40 & 1.77 \\
\hline
\end{tabular}

Notes. The transforms are specified in an abbreviated fashion, where "5a2" signifies "5 $a_{l m} \rightarrow$ map SHTs of spin 2", and "3m0" stands for "3 map $\rightarrow a_{l m}$ SHTs of spin 0". The runs were performed on a single CPU core.

There are several reasons for the obvious performance discrepancy between scalar and $s=2$ transforms. To a large part it is probably caused by the internal dependency chains of the $\lambda_{l m}$ recursion: even though the CPU could in principle start new operations every clock cycle, it has to wait for some preceding operations to finish first (which takes several cycles), because their results are needed in the next steps. When computing a recurrence, the CPU is therefore spending a significant fraction of time in a waiting state (see, e.g., Fog 2010). For the $s=2$ transform this is much less of an issue, since many more floating-point operations must be carried out, which are not interdependent, and the time spent on the $l$-recursion becomes subdominant.

Other reasons for the lower performance of the recursion are the necessity to deal with extended floating-point numbers and its rather high ratio of memory accesses compared to arithmetic operations.

\subsubsection{Gains by using simultaneous transforms}

The CPU time savings achievable by performing multiple SHTs together instead of one after another were measured on a HEALPix grid with $N_{\text {side }}=1024$ and $l_{\max }=2048$. Table 2 summarises the measured CPU times for an unsystematic selection of simultaneous and successive transforms, as well as the speedup.

The results show that for a large number of simultaneous SHTs the computation time can be cut roughly in half; this is the same asymptotic limit that also holds for SHTs with completely precomputed ${ }_{s} \lambda_{l m}(\vartheta)$ (see Sect. 3.1.3), but the presented approach has dramatically lower memory needs and can therefore be used for higher-resolution transforms. It is also evident that forward and backward transforms, as well as transforms of different spins, can be freely mixed while still preserving the performance increase.

\subsection{Memory consumption}

Figure 8 shows the maximum amount of memory allocated during a variety of SHTs carried out using libpsht and the Fortran HEALPix synfast facility. Since the memory required for forward and backward transforms is essentially equal, only one direction has been plotted. The sizes of SHTs with $l_{\max }<512$ could not be measured reliably due to their short running time and are therefore not shown. 


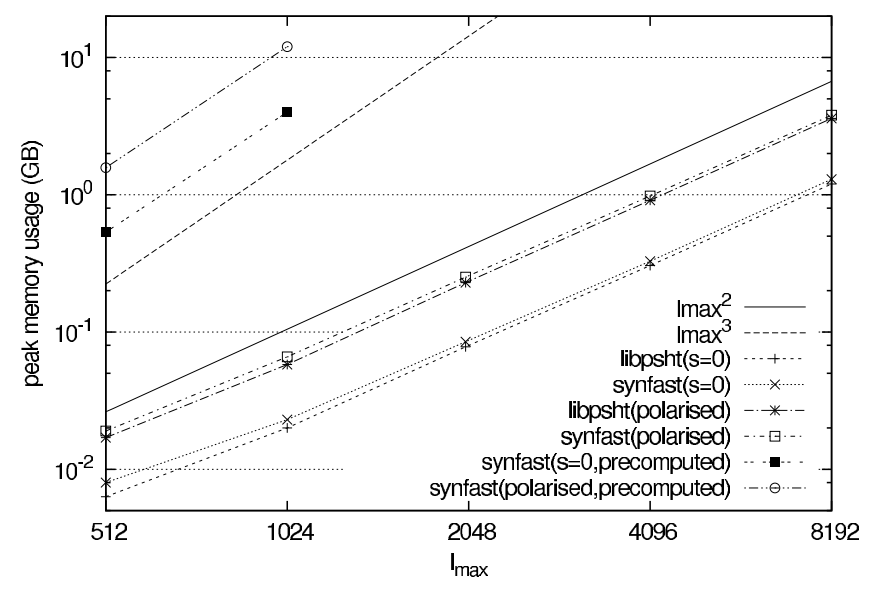

Fig. 8. Memory consumption of various SHTs performed with libpsht and synfast on a HEALPix grid with $N_{\text {side }}=l_{\max } / 2$. Input and output data are stored in single-precision format.

As can be seen, libpsht needs slightly less memory than synfast for equivalent operations; overall, the scaling is very close to the expected $l_{\max }^{2}$ if no precomputed ${ }_{s} \lambda_{l m}(\vartheta)$ are used. For comparison purposes, a few data points for synfast runs using precomputed scalar and tensor ${ }_{s} \lambda_{l m}(\vartheta)$ were also plotted; they clearly indicate the extremely high memory usage of these transforms, which scales with $l_{\max }^{3}$.

During the tests with multiple simultaneous threads it was observed that increasing the number of cores does not have a significant influence on the total required memory; for the concrete test discussed in Sect. 5.2.4, memory usage for the run with 16 threads was only $2 \%$ larger than for the single-threaded run.

\section{Conclusions}

Looking back at the goals outlined in Sect. 2.2 and the results of the benchmark computations above, it can be concluded that the current version of the libpsht package meets almost all specified requirements. It implements SHTs that have the same or a higher degree of accuracy as the other available implementations, can work on all spherical grids relevant to CMB studies, and is written in standard $\mathrm{C}$, which is very widely supported. The employed algorithms are significantly more efficient than those published and used in this field of research before; their memory usage is economic and allows transforms whose input and output data occupy almost all available space. When appropriate, vector capabilities of the CPUs, as well as shared memory parallelism are exploited, resulting in further performance gains.

Despite this range of capabilities, the package only consists of approximately 7000 lines of code, including the FFT implementation and in-line documentation for developers; except for a $\mathrm{C}$ compiler, it has no external dependencies.
Libpsht has been integrated into the simulation package of the Planck mission (Level-S, Reinecke et al. 2006), where it is interfaced with a development version of the $\mathrm{C}++$ HEALPix package $^{12}$ and a Fortran90 code performing SHTs on detector beam patterns; this demonstrates the feasibility of interfacing the library with $\mathrm{C}++$ and Fortran code.

There are two areas in which libpsht's functionality should probably be extended: it currently does not provide transforms for spins larger than 2, and there is no active support for distributing SHTs over several independent computing nodes. As was mentioned in Sect. 3.1.2, addressing the first point is probably not too difficult, and enhancing the library with ${ }_{s} Y_{l m}$ generators based on Wigner $d$ matrix elements is planned for one of the next releases. Regarding the second point, a combination of libpsht's central SHT functionality with the parallelisation strategy implemented by Radek Stompor's s2hat library appears very promising and is currently being investigated.

Libpsht is distributed under the terms of the GNU General Public License (GPL) version 2 (or later versions at the user's choice); the most recent version, alongside online technical documentation can be obtained at the URL http: // sourceforge. net/projects/libpsht/.

Acknowledgements. Some of the results in this paper have been derived using the HEALPix (Górski et al. 2005) and GLESP (Doroshkevich et al. 2005) packages. M.R. is supported by the German Aeronautics Center and Space Agency (DLR), under program 50-OP-0901, funded by the Federal Ministry of Economics and Technology. I am grateful to Volker Springel, Torsten Ensslin, Jörg Rachen and Georg Robbers for fruitful discussions and valuable feedback on drafts of this paper.

\section{References}

Bluestein, L. I. 1968, Northeast Electronics Research and Engineering Meeting Record, 10, 218

Crittenden, R. G., \& Turok, N. G. 1998 [arXiv:astro-ph/9806374], unpublished

Doroshkevich, A. G., Naselsky, P. D., Verkhodanov, O. V., et al. 2005, Int. J. Mod. Phys. D, 14, 275

Drepper, U. 2007, What Every Programmer Should Know About Memory, http: //www . akkadia.org/drepper/cpumemory .pdf

Driscoll, J. R., \& Healy, D. M. 1994, Adv. Appl. Math., 15, 202

Fog, A. 2010, Optimizing software in $\mathrm{C}++$, http://agner.org/optimize/ optimizing_cpp.pdf

Frigo, M., \& Johnson, S. G. 2005, Proc. IEEE, 93, 216, http://fftw.org/ fftw-paper-iee. pdf

Górski, K. M., Hivon, E., Banday, A. J., et al. 2005, ApJ, 622, 759

Huffenberger, K. M., \& Wandelt, B. D. 2010, ApJS, 189, 255

Kostelec, P., \& Rockmore, D. 2008, J. Fourier Analysis and Applications, 14, 145

Lewis, A. 2005, Phys. Rev. D, 71, 083008

Prézeau, G., \& Reinecke, M. 2010, ApJS, 190, 267

Reinecke, M., Dolag, K., Hell, R., Bartelmann, M., \& Enßlin, T. A. 2006, A\&A, 445,373

Swarztrauber, P. 1982, Vectorizing the Fast Fourier Transforms (New York: Academic Press), 51

Varshalovich, D. A., Moskalev, A. N., \& Khersonskii, V. K. 1988, Quantum Theory of Angular Momentum (World Scientific Publishers)

Wiaux, Y., Jacques, L., \& Vandergheynst, P. 2007, J. Comput. Phys., 226, 2359

12 The next major release of HEALPix $\mathrm{C}++$ will use libpsht. 